\title{
Fano resonance in a cavity-reflector hybrid system
}

\author{
Chengyu Yan, ${ }^{1,2, *}$ Sanjeev Kumar,,${ }^{1,2}$ Michael Pepper, ${ }^{1,2}$ Patrick See, ${ }^{3}$ Ian Farrer,${ }^{4, \dagger}$ \\ David Ritchie, ${ }^{4}$ Jonathan Griffiths, ${ }^{4}$ and Geraint Jones ${ }^{4}$ \\ ${ }^{1}$ London Centre for Nanotechnology, 17-19 Gordon Street, London WC1H OAH, United Kingdom \\ ${ }^{2}$ Department of Electronic and Electrical Engineering, University College London, Torrington Place, London WC1E 7JE, United Kingdom \\ ${ }^{3}$ National Physical Laboratory, Hampton Road, Teddington, Middlesex TW11 OLW, United Kingdom \\ ${ }^{4}$ Cavendish Laboratory, J. J. Thomson Avenue, Cambridge CB3 OHE, United Kingdom
}

(Received 10 October 2016; revised manuscript received 1 December 2016; published 26 January 2017)

\begin{abstract}
We present the results of transport measurements in a hybrid system consisting of an arch-shaped quantum point contact (QPC) and a reflector; together, they form an electronic cavity in between them. On tuning the arch-QPC and the reflector, an asymmetric resonance peak in resistance is observed at the one-dimension to two-dimension transition. Moreover, a dip in resistance near the pinch-off of the QPC is found to be strongly dependent on the reflector voltage. These two structures fit very well with the Fano line shape. The Fano resonance was found to get weakened on applying a transverse magnetic field, and smeared out at $100 \mathrm{mT}$. In addition, the Fano-like shape exhibited a strong temperature dependence and gradually smeared out when the temperature was increased from 1.5 to $20 \mathrm{~K}$. The results might be useful in realizing devices for quantum information processing.
\end{abstract}

DOI: 10.1103/PhysRevB.95.041407

There is a growing interest in realizing the electrical analog of photonic-cavity devices in condensed matter as they form the fundamental basis of tomorrow's quantum technologies for realizing the building blocks for quantum information processing and quantum circuits. Much success with photonic-cavity [1-4] based systems could be attributed to a better control over manipulating photons and achieving entanglement over a larger distance for information processing [5-8]. Electronic devices have not enjoyed a similar level of success due to limited advancements in controlling the quantum states of the electrons. Recently, an electronic cavity coupled to a quantum dot was demonstrated with the observation of spin coherent state in the regime of dot-cavity coupling due to the Kondo effect [9]. There have been experimental attempts in the past in coupling an electronic cavity with one-dimensional (1D) electrons using a quantum point contact (QPC) [10,11], however, in such cases the oscillations in the conductance [12-15] were primarily due to quantum interference rather than coupling between the $1 \mathrm{D}$-cavity states. Therefore, direct evidence of coupling between the 1D states of a QPC and the cavity states would be crucial in creating a platform for realizing cavity based tunable electronic devices.

In the present Rapid Communication, we demonstrate a cavity-reflector hybrid quantum device consisting of a QPC as the source of $1 \mathrm{D}$ electrons and an electronic cavity of twodimensional (2D) electrons, and show that when these states couple, they give rise to a Fano resonance [16].

The samples studied in the present work consist of a pair of arch-shaped gates, with QPC forming in the center of the arch,

\footnotetext{
*uceeya3@ucl.ac.uk
}

†Present address: Department of Electronic and Electrical Engineering, The University of Sheffield, Mappin Street, Sheffield S1 3JD, United Kingdom.

Published by the American Physical Society under the terms of the Creative Commons Attribution 3.0 License. Further distribution of this work must maintain attribution to the author(s) and the published article's title, journal citation, and DOI. and a reflector inclined at $75^{\circ}$ against the current flow direction, such that the center of the QPC and the reflector are aligned, as shown in Fig. 1(a) [17]. With this geometry, the interference between incident and reflected electrons which contribute to oscillations reported previously $[13,18,19]$ is significantly reduced, because the incident and reflected electrons are spatially separated, and thus all the features observed here are due to the coupling effect. The hybrid devices were fabricated on a high mobility two-dimensional electron gas (2DEG) formed at the interface of a GaAs $/ \mathrm{Al}_{0.33} \mathrm{Ga}_{0.67}$ As heterostructure. The 2DEG is situated $90 \mathrm{~nm}$ from the surface where the gates are deposited. The electron density (mobility) measured at $1.5 \mathrm{~K}$ was $1.80 \times 10^{11} \mathrm{~cm}^{-2}\left(2.1 \times 10^{6} \mathrm{~cm}^{2} \mathrm{~V}^{-1} \mathrm{~s}^{-1}\right)$, therefore, both the mean free path and phase coherence length were over $10 \mu \mathrm{m}$, which is much larger than the distance between the QPC and the reflector $(1.5 \mu \mathrm{m})$. All the measurements were performed with the standard lock-in technique in a cryofree pulsed-tube cryostat with a base temperature of $1.5 \mathrm{~K}$. For the four-terminal resistance measurement, a $10 \mathrm{nA}$ at $77 \mathrm{~Hz}$ ac current was applied while an ac voltage of $10 \mu \mathrm{V}$ at $77 \mathrm{~Hz}$ was used for the two-terminal conductance measurement. Figure 1(b) shows the conductance plot of the QPC with well-defined conductance plateaus; on the other hand, the conductance of the reflector drops, when measured separately, around $-0.2 \mathrm{~V}$, which indicates a sharp change in the transmission probability [Fig. 1(b), inset]. The three regimes identified from the characteristic of the reflector are labeled as regimes $1-3$, as shown in the inset.

To highlight the effect of the cavity states, we studied a nonlocal four-terminal resistance $R_{12,34}=V_{34} / I_{12}$ (hereafter denoted as $R$ ), as shown in Fig. 2. This measurement configuration allows one to study the interference between reflected electrons which propagate towards Ohmic 3 directly without being affected by the cavity states, and those go through the cavity and get modulated.

In regime 1 , where the reflector voltage $V_{r}$ was swept from -0.30 (top trace) to $-0.27 \mathrm{~V}$ (bottom trace), a pronounced asymmetric resonance structure, which shows a peak at a more negative arch-QPC voltage $V_{a}$ and a valley at the less negative 

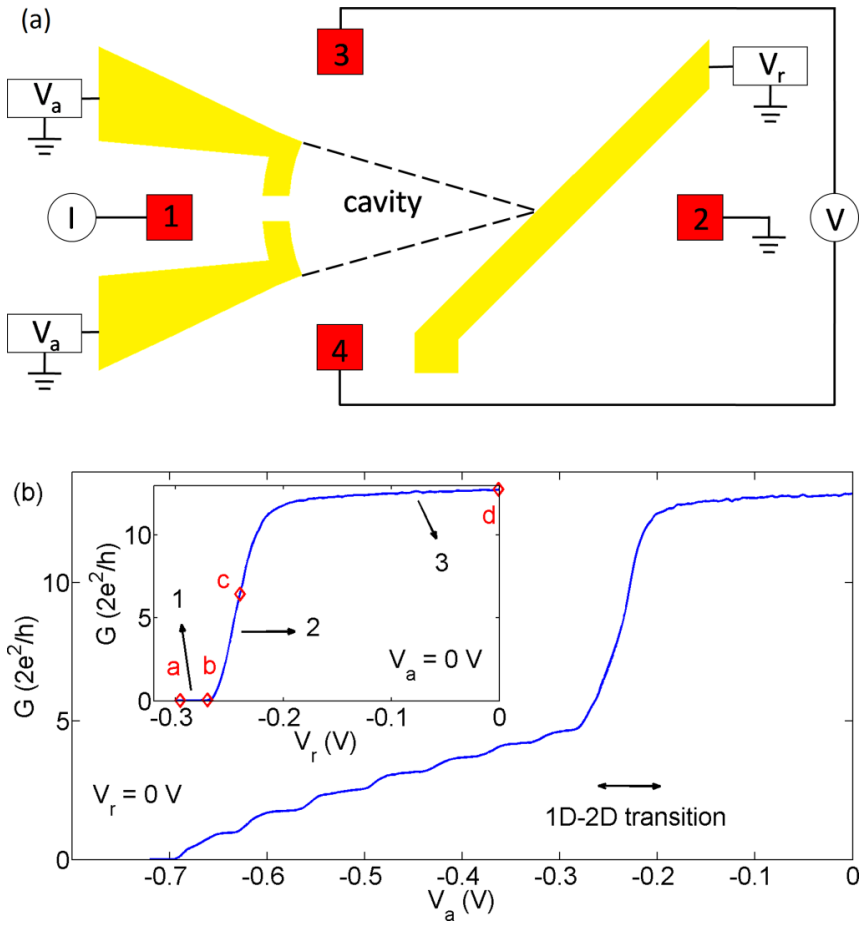

FIG. 1. Schematic of the device and the experiment setup. (a) The yellow blocks are metallic gates and red squares are Ohmics; excitation current is fed to Ohmic 1 while 2 is grounded; Ohmics 3 and 4 are voltage probes. The opening angle of the arch is $45^{\circ}$ and the radius (also the distance between the arch gate and reflector) is $1.5 \mu \mathrm{m}$, both the length and width of the QPC embedded in the arch is $200 \mathrm{~nm}$, the length of the inclined surface of the reflector is $3.0 \mu \mathrm{m}$, and the width is $300 \mathrm{~nm}$. (b) Differential conductance measurement of the QPC (main plot) and the reflector (inset). To be noted that in this plot when the QPC was measured, the reflector was grounded, and vice versa. The voltage applied to the QPC (reflector) was $V_{a}\left(V_{r}\right)$. Three regimes are identified from the reflector voltage characteristic and labeled as regime 1 (a-b), regime 2 (b-c), and regime 3 (c-d).

end, centered around $V_{a}=-0.2 \mathrm{~V}$, was observed, which is also the center of the 1D-to-2D transition regime of arch-QPC [as shown by a dotted rectangular box in Fig. 2(a)].

When the reflector was set to regime $2, V_{r}$ increases from -0.27 to $-0.24 \mathrm{~V}$ [Fig. 2(b)], the asymmetric resonance structure slowly evolves into a broad shoulder structure, and a dip gradually forms at the pinch-off regime of the QPC. It is interesting to notice that the dip starts forming when the asymmetric resonance structure fully smears out. In addition, the center of the shoulder structure shifts towards a more negative $V_{a}$ with increasing $V_{r}$ (see the solid line), and, on the contrary, the dip structure moves to a less negative $V_{a}$ (see the dashed line).

In regime $3\left(-0.24 \mathrm{~V} \leqslant V_{a} \leqslant 0\right)$, the intensity of both the shoulder structure and the dip decreases with less negative reflector voltage and finally smears out [Fig. 2(c)].

The asymmetric resonance structure observed at the $1 \mathrm{D}-2 \mathrm{D}$ transition regime and the dip structure in the pinch-off regime seem to arise from a dramatic change in the density of states (DOS) in the QPC in these regimes. However, such changes in DOS are independent of $V_{r}$ while the resonance and the dip structures are highly sensitive to $V_{r}$. In the present device,
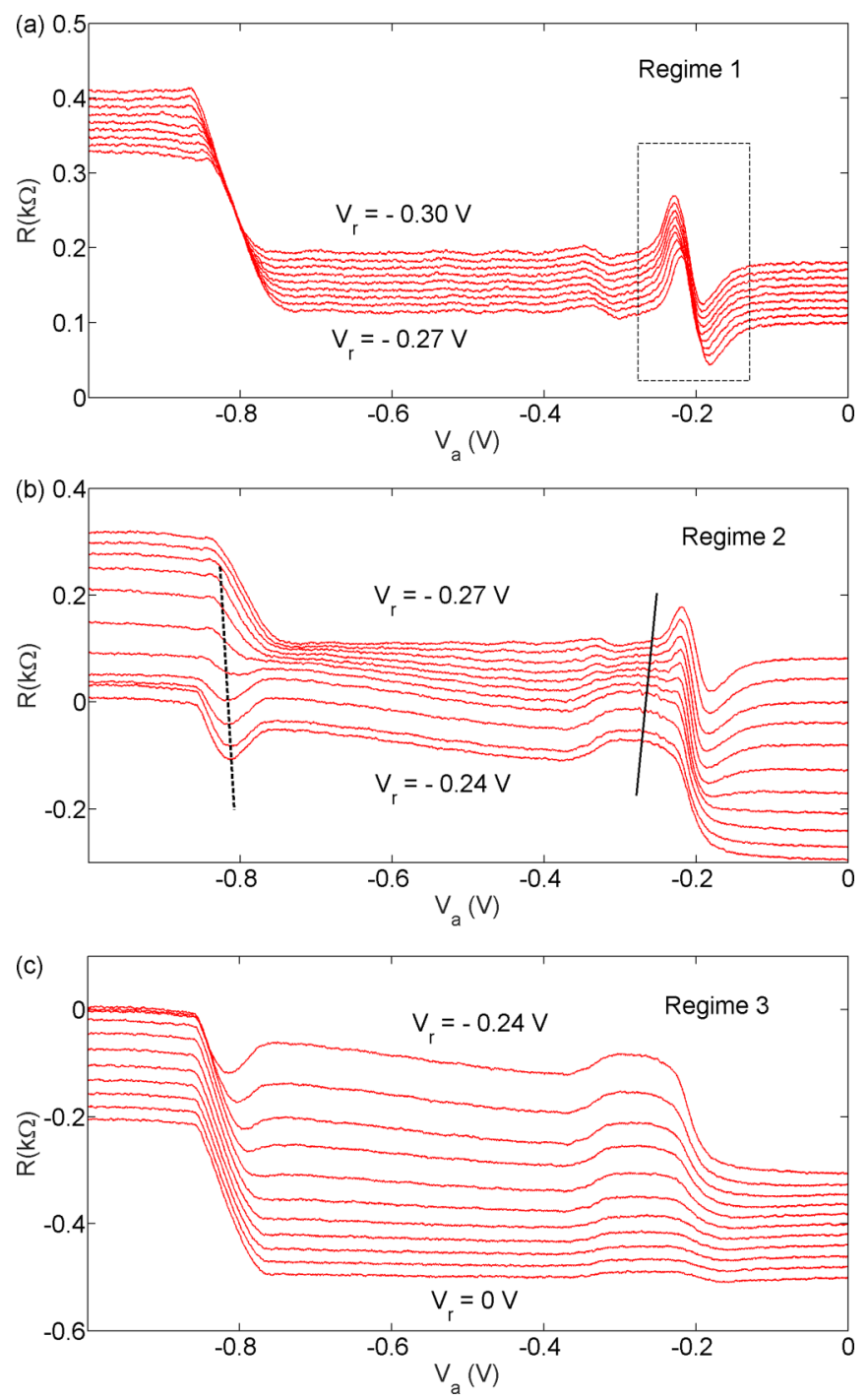

FIG. 2. $R$ as a function of arch-QPC voltage for various $V_{r}$. (a) Result in regime 1: A strong asymmetric resonance structure highlighted by a dotted rectangle occurs around -0.2 V. (b) Result in regime 2: The resonance structure slowly evolves into a broad shoulder structure (marked by the solid line), meanwhile, a dip gradually forms at the pinch-off regime of the arch-QPC (marked by the dashed line). (c) Result in regime 3: Intensity of both the shoulder structure and the dip decreases with less negative reflector voltage. Data have been offset vertically by $10 \Omega$ in each plot for clarity.

the angle of the reflector is properly designed to avoid back reflection of the electrons, so that it is unlikely that the reflected electrons would enter the QPC and perturb the DOS directly.

A similar dip structure was reported in a double-QPC experiment when the intruder QPC was swept into the pinch-off regime [20,21]; the effect was attributed to a Fano resonance which arises from the interference between the discrete states and the continuum [22], where the role of the intruder was to provide the continuum. In our system, the electronic cavity, which is defined using the arch gate and the reflector, having its states filled up to the chemical potential, hosts the continuum while the electrons injected from the arch-QPC are energetically discrete; thus the coupling between 

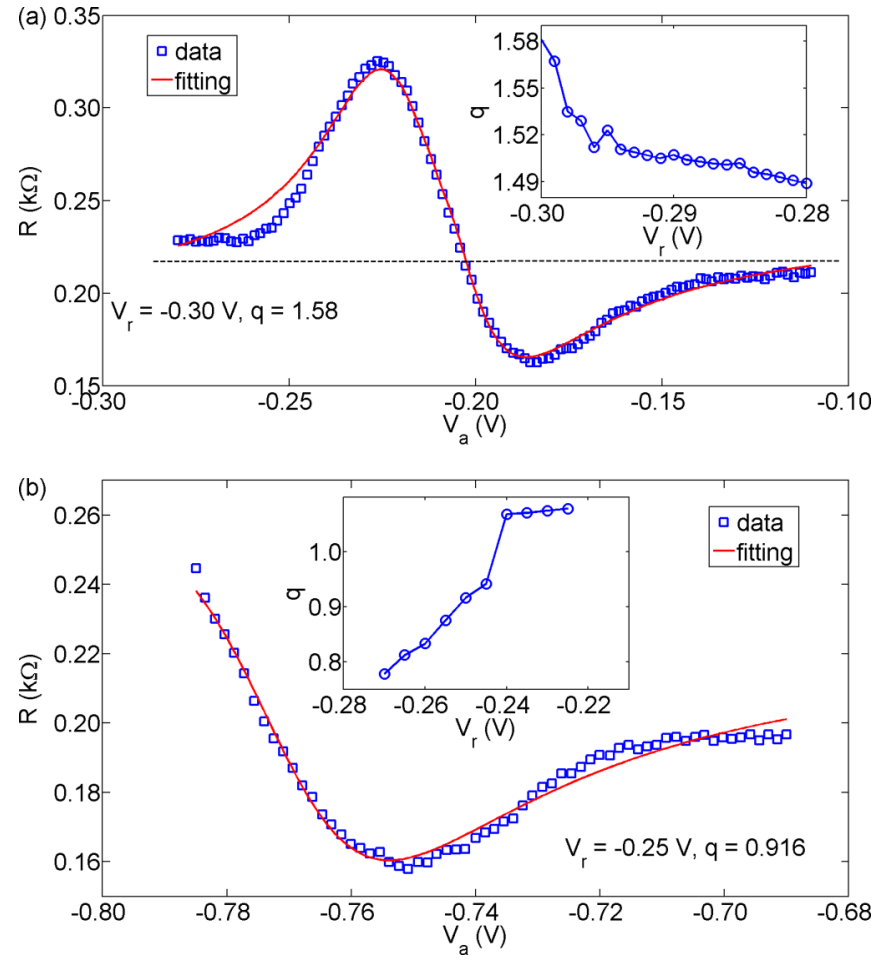

FIG. 3. Theoretical fitting of (a) the resonance and (b) the dip structures. It is remarkable that both the resonance structure $\left(V_{r}=-0.30 \mathrm{~V}\right)$ and the $\operatorname{dip}\left(V_{r}=-0.25 \mathrm{~V}\right)$ follow a well-defined Fano line shape. The horizontal black dotted line is a guide to the eye, reflecting that the saturated values on the left end of the experimental data (the 1D regime of QPC) and that on the right (the $2 \mathrm{D}$ regime) do not align. This may be due to a dramatic variation in DOS at the 1D-2D transition. The inset in (a) and (b) shows the Fano factor $q$ as a function of $V_{r}$ in the range where the structures are observable.

the QPC and the cavity states results in a Fano resonance at the two regimes, (1) near the pinch-off and (2) at the 1D-2D transition. In addition, weak oscillations are observed in the resistance in the 1D regime of the arch-QPC [Fig. 2(a)], which could be a consequence of interference of $1 \mathrm{D}$ electrons with the cavity states. A detailed study of this regime will be published elsewhere [23].

Figure 3 shows the fitting of the asymmetric resonance and the dip structures. It is remarkable that both structures follow a well-defined Fano line shape [22,24],

$$
R=R_{0} \frac{\left[q+\gamma\left(V-V_{0}\right)\right]^{2}}{1+\gamma^{2}\left(V-V_{0}\right)^{2}}+R_{\mathrm{inc}},
$$

where $R$ is the measured resistance, $R_{0}$ is a constant representing the amplitude of the resonance, $q$ is the Fano factor which decides the asymmetry of the line shape, $\gamma=20 \mathrm{~V}^{-1}$ (estimated from the Fermi energy and the pinch-off voltage), $V_{0}$ is the arch-gate voltage at the center of the resonance (dip), and $R_{\text {inc }}$ denotes the intrinsic contribution from the background [24]. However, it is noted that the fitting matches with the experimental result in the center while it diverges at both ends for Figs. 3(a) and 3(b), which is likely due to the fact that $R$ changes as a function of $V_{a}$ even in the absence of a quantum correction, because electrons are collimated when
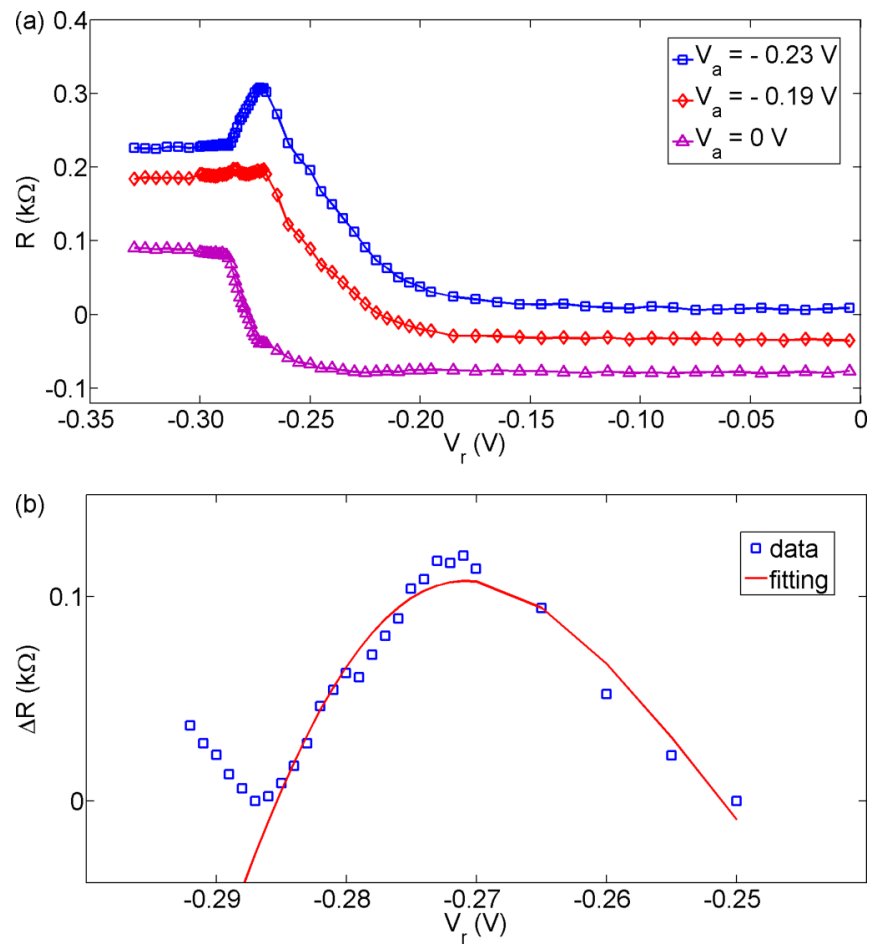

FIG. 4. $R$ as a function of reflector voltage for various $V_{a}$. (a) The arch-gate voltage was fixed at $-0.23 \mathrm{~V}(\square),-0.19 \mathrm{~V}(\diamond)$, and $0 \mathrm{~V}(\triangle)$, respectively, while sweeping the reflector voltage; data have been offset vertically by $50 \Omega$ for clarity. (b) Theoretical fitting of $\Delta R\left(V_{r}, V_{a}\right)=R\left(V_{r}, V_{a}\right)-R\left(V_{r}, 0\right)$, where $V_{a}=-0.23 \mathrm{~V}$, and using Eq. (1), where $q=1.47$, it is clear that the data follow a Fano line shape.

the QPC is operating in the $1 \mathrm{D}$ regime, whereas they spread out when the QPC is in the 2D regime [25]. Moreover, the divergence in the pinch-off regime is due to the fact that once the $1 \mathrm{D}$ channel is pinched off, the detected signal will not change any further. The inset in Figs. 3(a) and 3(b) shows the Fano factor $q$ as a function of $V_{r}$. It was seen that $q$, which represents the coupling between the QPC and the cavity, changes rapidly in both regimes, which is most likely due to a sharp change in DOS of the QPC [20,21] in the 1D-2D transition and the pinch-off regime, respectively. Concerning the fitting in Fig. 3(b), the structure occurs in the pinch-off regime where making $V_{a}$ even more negative will not change the signal, therefore, this leaves an open question as to whether the data at the pinch-off are the dip of the Fano resonance or do they follow a Breit-Wigner line shape. It is to be noted that an asymmetric Fano line shape is observed whenever resonant and nonresonant scattering paths interfere. However, when there is no interference between them, a symmetric Breit-Wigner resonance is expected [24]. If we look at Fig. 2(b) and focus on the dip in resistance at $V_{a}=-0.8 \mathrm{~V}$ as a function of $V_{r}$, it seems that the dip structure takes a symmetric shape before finally settling at $V_{r}=-0.24 \mathrm{~V}$ to reflect an asymmetric line shape. It may be possible that the lower dip at the pinch-off is moving through the Breit-Wigner line shape to the Fano line shape, depending on the coupling factor $q$. However, such observations need a detailed study and it is difficult to comment on them at the moment. 

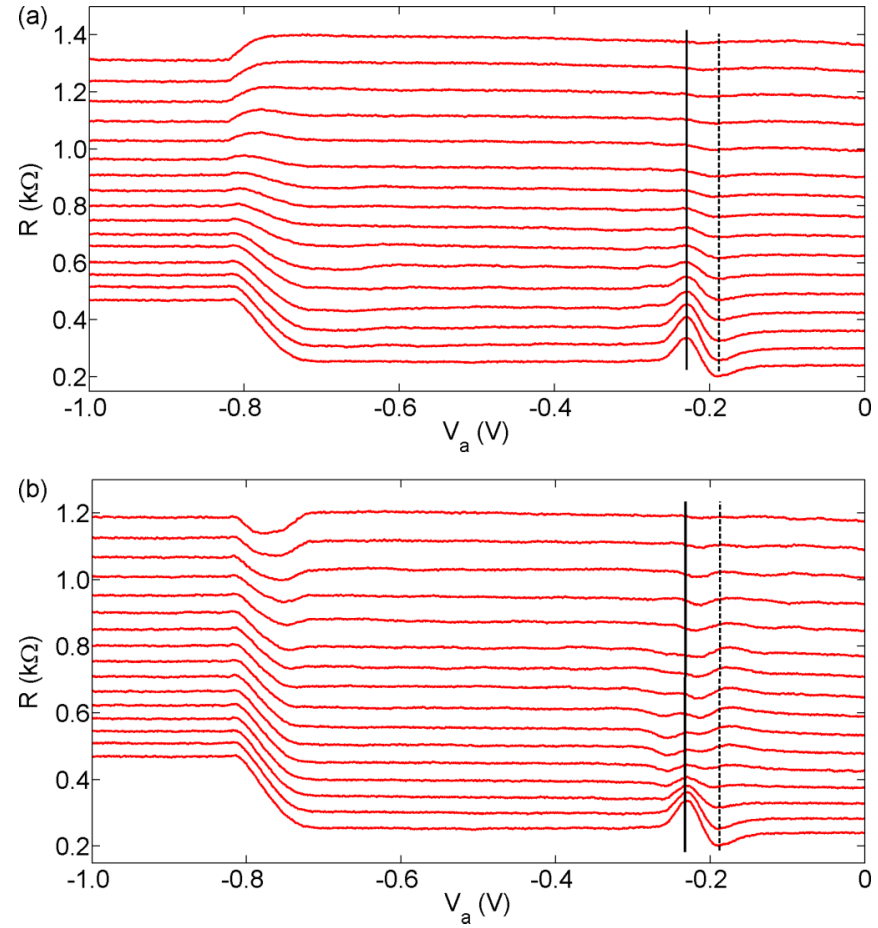

FIG. 5. Effect of perpendicular magnetic field. In (a) and (b) the transverse magnetic field is incremented from 0 (bottom trace) to $\pm 150 \mathrm{mT}$ (top trace), respectively, and it is seen that both the peak (marked by the solid line) and the dip (highlighted by the dashed line) rapidly weaken against the field, almost smearing out at $\pm 100 \mathrm{mT}$. Data have been offset vertically by $20 \Omega$ for clarity.

The Fano line shape arising from the coupling between the QPC and the cavity states can be modulated by either tuning the QPC states while fixing the cavity states, as already shown in Fig. 2, or in a complementary way by adjusting the cavity states and holding the QPC states as shown in Fig. 4(a). In this measurement, the reflector voltage was swept, which allows one to control the energy spacing of the cavity states while $V_{a}$ was set to -0.23 (the peak of the asymmetric resonance structure), -0.19 (the dip of the asymmetric resonance structure), and $0 \mathrm{~V}$, respectively, as shown in Fig. 4(a). When $V_{a}$ was set to -0.19 and $0 \mathrm{~V}$, respectively, where the cavity was not defined, it was seen that the resistance $R$ was initially almost constant when the reflector voltage $V_{r}>-0.20 \mathrm{~V}$. On decreasing $V_{r}<-0.20 \mathrm{~V}$, a rise in resistance occurs simultaneously when the reflector conductance drops, where the reflection probability $r$ increases rapidly, and then $R$ saturates when $r$ becomes unity [see the inset in Fig. 1(b)]. When $V_{a}$ was set to $-0.23 \mathrm{~V}, R$ follows a similar trend as the previous case when $V_{r}>-0.20 \mathrm{~V}$, where the cavity was not activated. After the cavity was switched on, an anomalous peak in $R$ occurs when $-0.28 \mathrm{~V} \leqslant V_{r} \leqslant-0.25 \mathrm{~V}$. The relative peak intensity, $\Delta R\left(V_{r}, V_{a}\right)=R\left(V_{r}, V_{a}\right)-R\left(V_{r}, 0\right)$, fits very well with the Fano line shape, as shown in Fig. 4(b) using Eq. (1), here $R\left(V_{r}, 0\right)$ is taken as the background signal because it accounts for the change in $R$ due to the change in reflection probability only, and it is always present as the background signal regardless of $V_{a}$, and the resonance structure superposes on such a background; therefore, to highlight the resonance, we subtracted the background.
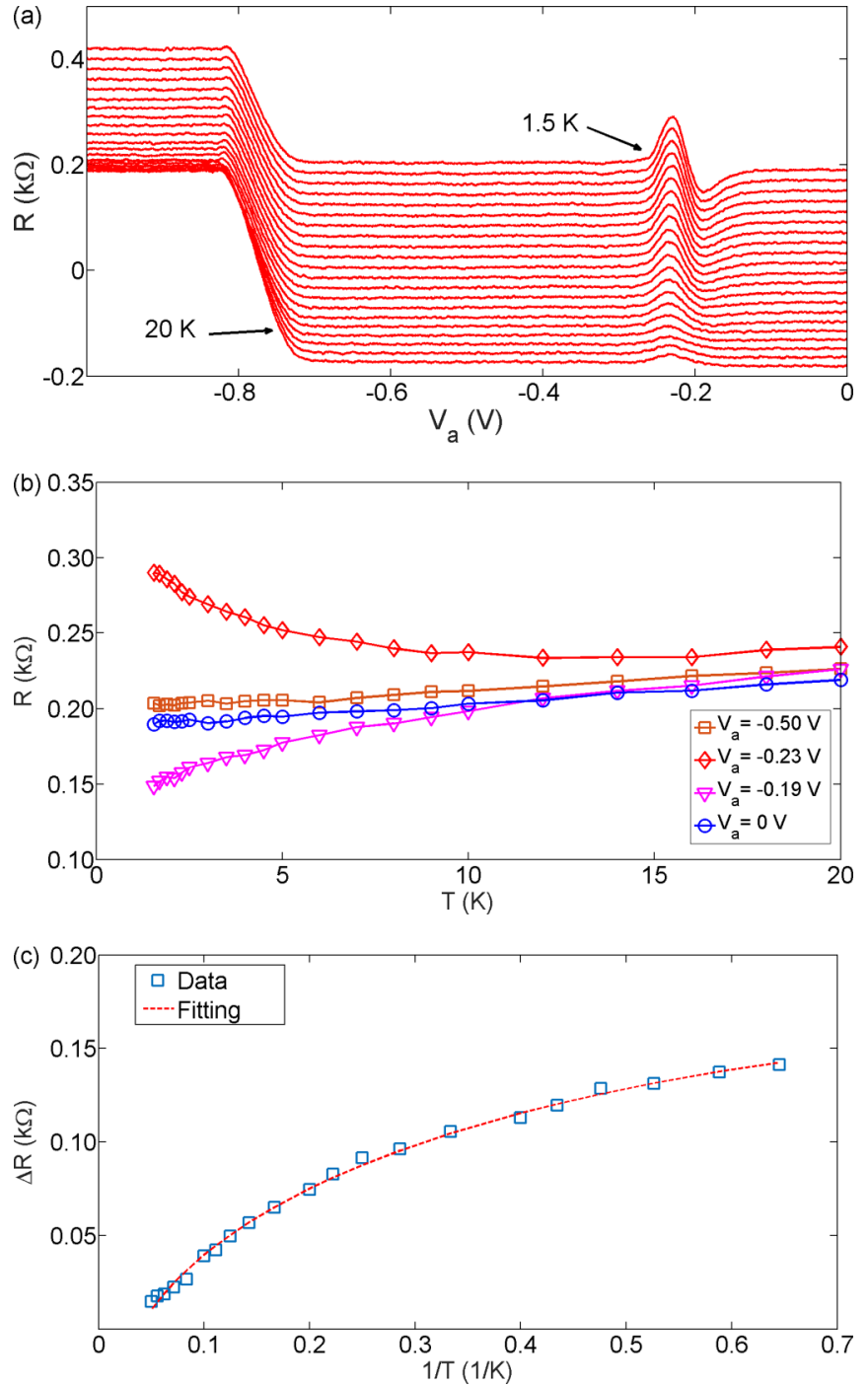

FIG. 6. Temperature dependence of $R$. (a) $R$ in regime $1\left(V_{r}=\right.$ $-0.3 \mathrm{~V}$ ) as a function of temperature from $1.5 \mathrm{~K}$ (top trace) to $20 \mathrm{~K}$ (bottom trace): It may be seen that the intensity of both the peak and the dip structures decreases with increasing temperature. Data have been offset vertically by $20 \Omega$ for clarity. (b) The temperature dependence of $R$ for $V_{a}=-0.50(\square),-0.23(\diamond),-0.19(\nabla)$, and $0 \mathrm{~V}(\bigcirc)$; solid lines are a guide to the eye. (c) Mott fitting for the temperature dependence of the peak structure, $\Delta R\left(V_{a}, T\right)=$ $R\left(V_{a}, T\right)-R(0, T)$, where $V_{a}=-0.23 \mathrm{~V}, R_{1}=-0.2656 \mathrm{k} \Omega, R_{2}=$ $0.4238 k \Omega, T_{0}=1.2 \mathrm{~K}$.

Coupling and decoupling between discrete and continuum states lead to the Fano resonance, thus the parameters which affect the coupling, apart from electrostatic confinement, are expected to dramatically influence the line shape [21]. Here, we present the effect of both positive and negative transverse magnetic fields, as shown in Figs. 5(a) and 5(b), respectively. The intensity of the asymmetric resonance structure is highly sensitive to the magnetic field in both field orientations, and almost smears out at a field of $\pm 100 \mathrm{mT}$. The reason for the reduction in the intensity against the magnetic field is threefold: First, the chance of an electron entering the cavity is magnetic field dependent; second, the cavity states, which 
represent the quantization of standing waves in the cavity, are highly dependent on the trajectory of electrons, and with a large magnetic field the electrons in the cavity become more localized, and therefore coupling between 1D and cavity states is weakened; and, third, the perpendicular magnetic field also leads to a reduction of phase coherence length and thereby weakens the interference effect [26,27]. A slight difference in the line shape for negative and positive fields is likely to arise from the fact that both the inclined reflector and the negative field favor Ohmic 3, while a positive field prefers guiding electrons to Ohmic 4.

We studied the temperature dependence of $R$ as shown in Fig. 6. The reflector voltage was set to $-0.3 \mathrm{~V}$, where both the peak and dip of the asymmetric resonance structure are pronounced. It is found that the intensity of the peak and the dip decreases against increasing temperature, and the dip structure smears out at around $16 \mathrm{~K}$, while the peak survives up to $20 \mathrm{~K}$ (although the height drops significantly). The evolution of the line shape against temperature is clearer in Fig. 6(b), and it is seen that $R$ increases slowly and almost linearly against temperature when $V_{a}$ is set to -0.50 or $0 \mathrm{~V}$, while the peak structure $\left(V_{a}=-0.23 \mathrm{~V}\right)$ and the dip structure $\left(V_{a}=\right.$ $-0.19 \mathrm{~V})$ change rapidly with increasing temperature. The fitting of the temperature dependence of the relative intensity of the peak structure, $\Delta R\left(V_{a}, T\right)=R\left(V_{a}, T\right)-R(0, T)$, where $V_{a}=-0.23 \mathrm{~V}$, resembles a Mott line shape [28] as shown in Fig. 6(c), using the relation

$$
R=R_{1} e^{\left(\frac{T_{0}}{T}\right)^{\frac{1}{2}}}+R_{1} e^{\left(\frac{T_{0}}{T}\right)^{\frac{1}{3}}},
$$

where $R_{1}, R_{2}$ are fitting parameters while $T_{0}$ is defined as

$$
T_{0} \propto \frac{1}{k_{B} N\left(E_{F}\right) \xi^{3}},
$$

where $k_{B}$ is the Boltzmann constant, $N\left(E_{F}\right)$ is the density of states in the absence of electron-electron interactions, $\xi$ is the localization length [29], and $T_{0}=1.2 \mathrm{~K}$ is extracted from the fitting. The $\frac{1}{2}$ and $\frac{1}{3}$ terms arise from the contribution of $1 \mathrm{D}$ and $2 \mathrm{D}$, respectively. This agrees with the fact that the Fano resonance is a direct manifestation of coupling between the QPC and the cavity states.

Mott's law was initially proposed for random hopping in disordered system [28], whereas clean systems generally do not follow Mott's law explicitly [e.g., see the trace for $V_{a}=0 \mathrm{~V}$ in Fig. 6(b)]. Random hopping accounts for a compromise between the spatial and energetic separations. It is possible that with the cavity switched on, the multiple reflection process is a mimic of the random hopping process, because the energy changing process in the cavity is accompanied with a spatial change [18] (states in the cavity are sensitive to the confinement length, and the confinement length, in turn, is coordinate sensitive).

In conclusion, we have shown the operation of a hybrid quantum device consisting of an arch-shaped QPC coupled to an electronic cavity, whose states can be tuned using a reflector gate. We have shown that, upon reducing the width of the QPC and increasing the reflection probability, Fano resonance can be produced at the 1D-2D transition and near the pinch-off regime. The Fano resonant structure is very sensitive to the transverse magnetic field, and exhibits a Mott line-shape temperature dependence. Such systems show the potential of hybrid devices in realizing tools for quantum information processing.

This work was supported by the Engineering and Physical Sciences Research Council (EPSRC), U.K.
[1] A. Rauschenbeutel, G. Nogues, S. Osnaghi, P. Bertet, M. Brune, J. M. Raimond, and S. Haroche, Phys. Rev. Lett. 83, 5166 (1999).

[2] K. Hennessy, A. Badolato, M. Winger, D. Gerace, M. Atatüre, S. Gulde, S. Fält, E. L. Hu, and A. Imamoğlu, Nature (London) 445, 896 (2007).

[3] T. Niemczyk, F. Deppe, H. Huebl, E. Menzel, F. Hocke, M. Schwarz, J. Garcia-Ripoll, D. Zueco, T. Hümmer, E. Solano et al., Nat. Phys. 6, 772 (2010).

[4] L.-M. Duan and H. J. Kimble, Phys. Rev. Lett. 92, 127902 (2004).

[5] P. G. Kwiat, K. Mattle, H. Weinfurter, A. Zeilinger, A. V. Sergienko, and Y. Shih, Phys. Rev. Lett. 75, 4337 (1995).

[6] T. Yoshie, A. Scherer, J. Hendrickson, G. Khitrova, H. Gibbs, G. Rupper, C. Ell, O. Shchekin, and D. Deppe, Nature (London) 432, 200 (2004)

[7] S. G. Carter, T. M. Sweeney, M. Kim, C. S. Kim, D. Solenov, S. E. Economou, T. L. Reinecke, L. Yang, A. S. Bracker, and D. Gammon, Nat. Photonics 7, 329 (2013).

[8] D. Englund, B. Shields, K. Rivoire, F. Hatami, J. Vuckovic, H. Park, and M. D. Lukin, Nano Lett. 10, 3922 (2010).

[9] C. Rössler, D. Oehri, O. Zilberberg, G. Blatter, M. Karalic, J. Pijnenburg, A. Hofmann, T. Ihn, K. Ensslin, C. Reichl, and W. Wegscheider, Phys. Rev. Lett. 115, 166603 (2015).
[10] D. A. Wharam, T. J. Thornton, R. Newbury, M. Pepper, H. Ahmed, J. E. F. Frost, D. G. Hasko, D. C. Peacock, D. A. Ritchie, and G. A. C. Jones, J. Phys. C 21, L209 (1988).

[11] B. J. van Wees, H. van Houten, C. W. J. Beenakker, J. G. Williamson, L. P. Kouwenhoven, D. van der Marel, and C. T. Foxon, Phys. Rev. Lett. 60, 848 (1988).

[12] M. Saito, T. Usuki, M. Okada, T. Futatsugi, R. A. Kiehl, and N. Yokoyama, Appl. Phys. Lett. 65, 3087 (1994).

[13] J. A. Katine, M. A. Eriksson, A. S. Adourian, R. M. Westervelt, J. D. Edwards, A. Lupu-Sax, E. J. Heller, K. L. Campman, and A. C. Gossard, Phys. Rev. Lett. 79, 4806 (1997).

[14] D. S. Duncan, M. A. Topinka, R. M. Westervelt, K. D. Maranowski, and A. C. Gossard, Phys. Rev. B 64, 033310 (2001).

[15] C. A. U. Díaz, J. Flores, and A. P. Ponce, Solid State Commun. 133, 93 (2005).

[16] In Ref. [9], the dot-cavity hybrid system is dominated by the Kondo effect. However, in the cavity-reflector hybrid quantum device presented here, consisting of a QPC and an electronic cavity, the Kondo effect is found to be absent, however, the device setup allows us to study the two-path interference effect which results in the Fano resonance, a direct evidence of coupling between the $1 \mathrm{D}$ and cavity states. 
[17] The present device provides control over the relevant parameters required to study the coupling effect, however, due to a limited number of gates, changes in the QPC states may be correlated with the cavity states. This limitation can be improved in future devices by patterning a local top gate over the QPC to adjust the QPC states without affecting the cavity states.

[18] J. S. Hersch, M. R. Haggerty, and E. J. Heller, Phys. Rev. Lett. 83, 5342 (1999).

[19] J. S. Hersch, M. R. Haggerty, and E. J. Heller, Phys. Rev. E 62, 4873 (2000).

[20] Y. Yoon, M.-G. Kang, T. Morimoto, L. Mourokh, N. Aoki, J. L. Reno, J. P. Bird, and Y. Ochiai, Phys. Rev. B 79, 121304 (2009).

[21] J. Fransson, M.-G. Kang, Y. Yoon, S. Xiao, Y. Ochiai, J. Reno, N. Aoki, and J. Bird, Nano Lett. 14, 788 (2014).

[22] U. Fano, Phys. Rev. 124, 1866 (1961).
[23] C. Yan, S. Kumar, M. Pepper, P. See, I. Farrer, D. Ritchie, J. Griffiths, and G. Jones (unpublished).

[24] J. Göres, D. Goldhaber-Gordon, S. Heemeyer, M. A. Kastner, H. Shtrikman, D. Mahalu, and U. Meirav, Phys. Rev. B 62, 2188 (2000).

[25] L. W. Molenkamp, A. A. M. Staring, C. W. J. Beenakker, R. Eppenga, C. E. Timmering, J. G. Williamson, C. J. P. M. Harmans, and C. T. Foxon, Phys. Rev. B 41, 1274 (1990).

[26] M. Kaveh, M. J. Uren, R. A. Davies, and M. Pepper, J. Phys. C 14, L413 (1981).

[27] S. McPhail, C. E. Yasin, A. R. Hamilton, M. Y. Simmons, E. H. Linfield, M. Pepper, and D. A. Ritchie, Phys. Rev. B 70, 245311 (2004).

[28] N. Mott, Philos. Mag. 19, 835 (1969).

[29] N. Mott and E. Davis, Electronic Processes in Noncrystalline Materials, 2nd ed. (Clarendon, Oxford, UK, 1979). 